\title{
Vigilancia de infecciones asociadas a la atención en salud, resistencia bacteriana y consumo de antibióticos en hospitales de alta complejidad, Colombia, 2011
}

\author{
Andrea Patricia Villalobos ${ }^{1,2}$, Liliana Isabel Barrero ${ }^{1,2}$, Sandra Milena Rivera ${ }^{1,2}$, \\ María Victoria Ovalle ${ }^{1,2}$, Danik Valera² \\ 1 Equipo Funcional Infecciones Asociadas a la Atención en Salud, Bogotá, D.C., Colombia \\ 2 Dirección de Vigilancia y Análisis del Riesgo en Salud Pública, Instituto Nacional de Salud, Bogotá, D.C., Colombia
}

Introducción. Prevenir las infecciones adquiridas en los hospitales, en especial las causadas por microorganismos resistentes, es una prioridad. Por esta razón, Colombia inició la vigilancia nacional de estos eventos realizando una prueba piloto.

Objetivo. Describir el comportamiento de las infecciones asociadas a dispositivos, resistencia bacteriana y consumo de antibióticos en instituciones con unidades de cuidados intensivos durante el 2011.

Materiales y métodos. Se llevó a cabo un estudio observacional descriptivo en 10 instituciones de salud de Antioquia, Valle del Cauca y Bogotá. Se diseñaron protocolos de vigilancia para cada evento, implementados por profesionales de salud entrenados. Se diseñó una herramienta en línea para la notificación y análisis de tasas de infección, porcentajes de utilización de dispositivos y dosis diarias definidas de antibióticos. Mediante el software Whonet 5.6 se reportaron y analizaron los perfiles y fenotipos de resistencia bacteriana.

Resultados. La infección del torrente sanguíneo fue la más frecuente (tasa mayor de 4,8 por 1.000 días-catéter), seguida de la neumonía asociada al respirador e infección urinaria asociada a catéter, con amplia variación entre instituciones. Se observó un mayor consumo de meropenem en las unidades de cuidados intensivos (dosis diarias definidas, 22,5 por 100 camas-día), resistencia elevada a carbapenémicos (>11,6\%) y cefalosporinas de tercera generación (>25,6\%) en enterobacterias en las unidades de cuidados intensivos y en otras áreas de hospitalización. El porcentaje de Staphylococcus aureus resistente a la meticilina fue mayor en otras áreas de hospitalización (34,3\%).

Conclusiones. Se trata de la primera aproximación integral a la problemática de estos eventos en Colombia. Es necesario implementar la vigilancia nacional que permita orientar acciones gubernamentales e institucionales para la prevención y control de infecciones, contención de la resistencia y promoción del uso prudente de antibióticos acompañados de un proceso de seguimiento y supervisión.

Palabras clave: vigilancia en salud pública, infección hospitalaria, control de infecciones, farmacorresistencia bacteriana, utilización de medicamentos, Colombia.

doi: http://dx.doi.org/10.7705/biomedica.v34i0.1698

Surveillance of healthcare associated infections, bacterial resistance and antibiotic consumption in high-complexity hospitals in Colombia, 2011

Introduction: Preventing healthcare associated infections, especially for resistant microorganisms, is a priority. In Colombia, the surveillance of such events was started through a national pilot study.

Objective: To describe the epidemiology of device-associated infections, bacterial resistance and antibiotic consumption patterns in institutions with intensive care units (ICU), 2011.

Materials and methods: Descriptive observational study in 10 health institutions from three Colombian provinces: Antioquia, Valle del Cauca, and Bogotá. Surveillance protocols were designed and implemented by trained health professionals in each hospital. A web tool was designed for data reporting and analysis. Infection rates, device-use percentages and antibiotics defined daily dose (DDD) were calculated. Bacterial resistance phenotypes and profiles were reported and analyzed using Whonet 5.6.

Results: The most common event was bloodstream infection (rate $>4.8 / 1000$ catheter-days) followed by ventilator-associated pneumonia (VAP) and catheter-related urinary tract infection, showing a wide

\section{Contribución de los autores:}

Andrea Patricia Villalobos, Liliana Isabel Barrero y Sandra Milena Rivera: diseño, gestión y ejecución del proyecto, recolección y análisis de los datos, redacción del manuscrito.

Danik Valera: diseño y gestión del proyecto.

María Victoria Ovalle: análisis de los datos, redacción del manuscrito.

Todos los autores participaron en la asesoría temática y metodológica y en la revisión del manuscrito. 
variability among institutions. A high consumption of meropenem in the ICU (DDD 22.5/100 bedsday) was observed, as well as a high carbapenem resistance $(>11.6 \%)$ and a high frequency of third generation cephalosporins resistance (>25.6\%) in Enterobacteriaceae in ICUs and hospitalization wards. The percentage of methicillin-resistant Staphylococcus aureus was higher in hospitalization wards (34.3\%).

Conclusions: This is the first experience in measuring these events in Colombia. It is necessary to implement a national surveillance system aimed at guiding governmental and institutional actions oriented to infection prevention and control, to resistance management and to the promotion of antibiotics rational use, along with a follow-up and monitoring process.

Key words: Public health surveillance, cross infection, infection control; drug resistance, bacterial; drug utilization, Colombia.

doi: http://dx.doi.org/10.7705/biomedica.v34i0.1698

Las infecciones asociadas a la atención en salud se definen como un proceso localizado o sistémico resultado de una reacción adversa a la presencia de un agente infeccioso o sus toxinas, que no estaba presente ni incubándose al ingreso de una institución y que cumple con una serie de criterios específicos (1). Este tipo de infecciones ocurren en cualquier ámbito de atención, incluidos los centros de atención ambulatoria, los sitios de cuidado crónico, los centros de rehabilitación y los hospitales (en donde se conocen como infecciones hospitalarias). Su importancia se reconoce por ser una de las causas de enfermedades y muerte potencialmente prevenibles $(2,3)$.

Los datos de la Organización Panamericana de la Salud (OPS) indican que más de 1,4 millones de personas en el mundo contraen infecciones en el hospital (4). En los países desarrollados, la prevalencia de pacientes hospitalizados que adquieren, al menos, una infección asociada a la atención en salud se encuentra entre 3,5 y $12 \%$, mientras que en los países en desarrollo varía entre 5,7 y 19,1\%, alcanzando en algunos de estos últimos países una proporción incluso mayor a $25 \%$ de pacientes afectados (5).

En los Estados Unidos y Europa este tipo de infecciones producen anualmente entre 99.000 y 110.000 muertes, millones de días de estancia hospitalaria adicionales $(1,5)$ y entre US $\$ 28.000$ y 33.000 millones de dólares en gastos de atención en salud que podrían evitarse (6).

\footnotetext{
Correspondencia:

Andrea Patricia Villalobos, Dirección de Vigilancia y Análisis del Riesgo en Salud Pública, Instituto Nacional de Salud, Av. Calle 26 N 51 -20, bloque B, segundo piso, Bogotá, D.C., Colombia Teléfono/fax: (571) 2207700 , extensión 1425

avillalobos@ins.gov.co, iaas.ins@gmail.com

Recibido: 07/06/13; aceptado: 15/10/13
}

El manejo de las infecciones asociadas a la atención en salud se hace más complejo si se contempla el uso inadecuado de los antimicrobianos a nivel hospitalario y comunitario, considerado hoy como el principal factor responsable de la emergencia y diseminación de microorganismos resistentes, lo cual se ha convertido en una seria amenaza para la salud pública a nivel mundial, sobre todo porque hay muy pocas opciones terapéuticas disponibles y una insuficiente inversión en investigación y desarrollo de nuevos fármacos (7-9).

El conocer la problemática en torno a estas infecciones constituye una necesidad de los gobiernos para poder orientar mejor las acciones de prevención y control. En este sentido, la vigilancia, componente clave de cualquier sistema de salud, permite identificar las oportunidades de prevención de estos eventos en el ámbito hospitalario y apoyar la mejora continua en la calidad de los resultados en salud (2).

En las Américas, algunos países han incorporado la vigilancia nacional de infecciones asociadas a la atención en salud y resistencia bacteriana en el ámbito hospitalario de manera satisfactoria, como es el caso de Argentina, Chile, Uruguay, Estados Unidos y Canadá; sin embargo, los objetivos, indicadores, definiciones y metodologías varían entre ellos (10-14).

En Latinoamérica, algunos países vigilan estos eventos en sus instituciones de salud, pero no tienen datos nacionales y otros aún no cuentan con una vigilancia estructurada ni en los servicios de salud ni en el nivel nacional $(15,16)$. Además, son pocos los que logran integrar la vigilancia de estas infecciones con la de la resistencia bacteriana y el uso de antimicrobianos (13).

En Colombia, existen iniciativas que han aportado al conocimiento del problema de las infecciones asociadas a la atención en salud y la resistencia 
bacteriana, aunque de manera sectorizada; sin embargo, con los datos de algunas instituciones de salud, redes de vigilancia y grupos de investigación del país, se observa que la situación de estas infecciones y la resistencia bacteriana no se aleja de lo reportado por otros países de la región (17-19).

En este contexto, en el nivel nacional se propuso implementar la vigilancia de las infecciones asociadas a la atención en salud, inicialmente las infecciones asociadas a dispositivos, la resistencia bacteriana y el consumo de antibióticos, este último a manera de primera aproximación al conocimiento del uso de los antibióticos que permita abordar el problema desde diferentes perspectivas relacionadas con el aumento de la resistencia bacteriana, la elevada carga de infecciones y el uso inapropiado de antibióticos en los hospitales; por lo tanto, el presente estudio se fijó el objetivo de identificar el comportamiento de estos tres eventos en instituciones de alta complejidad a partir de los resultados de la fase de implementación de una prueba piloto nacional en 2011.

\section{Materiales y métodos}

Se llevó a cabo un estudio observacional descriptivo y prospectivo en 10 instituciones de salud, públicas y privadas, de alta complejidad de Antioquia y Valle del Cauca y de Bogotá, que contaban con unidad de cuidados intensivos y participaban en un programa de control de calidad externo en microbiología. Las instituciones fueron seleccionadas mediante un muestreo por conveniencia, y manifestaron por escrito su intención de participar; se tuvo en cuenta que los departamentos seleccionados presentaban el mayor volumen de instituciones de salud del país.

La prueba piloto se desarrolló en cuatro fases: preparación, implementación, evaluación y retroalimentación. La fase de preparación (julio de 2010 a abril de 2011), incluyó la revisión de la literatura y de las experiencias de otros sistemas de vigilancia de las infecciones asociadas a la atención en salud, la resistencia bacteriana y el consumo de antibióticos a nivel nacional e internacional que respaldaron el diseño de tres protocolos de vigilancia y de la herramienta en línea para la notificación de estos eventos.

Se diseñó y ejecutó un consenso formal de expertos (Delphi en línea) (20) para la definición de las estrategias de vigilancia, microorganismos, fenotipos de resistencia y antibióticos por vigilar, el cual involucró a representantes de sociedades científicas, universidades, grupos de investigación e instituciones de salud de alta complejidad. Los documentos finales fueron revisados por expertos nacionales e internacionales. En esta fase se formalizó la inclusión de las entidades territoriales y de las instituciones de salud, seguida de un proceso de sensibilización, diseminación y estandarización de la estrategia de vigilancia destinada a las personas involucradas en la vigilancia de estos eventos.

La fase de implementación se llevó a cabo entre mayo de 2011 y marzo de 2012, periodo en el cual se recolectaron y notificaron los datos colectivos de la vigilancia mensual desde mayo hasta diciembre de 2011. La fase de evaluación (septiembre y octubre de 2011), incluyó la evaluación interna y la externa, realizadas por el Instituto Nacional de Salud y la OPS, respectivamente. Finalmente, la fase de retroalimentación se concibió como un proceso transversal que incluyó la asistencia técnica (presencial, electrónica y telefónica), los informes, las mesas de trabajo y la divulgación.

\section{Vigilancia de infecciones asociadas a dispositivos}

Esta se hizo de manera activa y permanente en las unidades de cuidados intensivos de adultos, pediátricas y neonatales. La caracterización y seguimiento de los casos de neumonía asociada a respiración mecánica asistida, de infección del torrente sanguíneo asociada a catéter central y de infección sintomática del tracto urinario asociada a catéter urinario estuvo a cargo de personal de salud entrenado en la aplicación de las definiciones de vigilancia de la National Healthcare Safety Network de los Centers for Disease Control and Prevention (NSHN/CDC) traducidas y adaptadas en el 2010 (21). No se vigilaron las infecciones sintomáticas del tracto urinario asociadas a catéter urinario en neonatos.

La recolección de información para el cálculo de las tasas de incidencia de las infecciones asociadas a dispositivos y el porcentaje de uso de dispositivos estuvo a cargo de un profesional de enfermería capacitado en la búsqueda diaria de casos de infección (numerador) y de pacientes con respirador mecánico, catéter central, catéter urinario en unidad de cuidados intensivos y días-paciente (denominadores); esta tarea se complementó con la revisión de las historias clínicas, registros de enfermería, registros de signos vitales, órdenes médicas de prescripción de antibióticos y reportes de cultivos microbiológicos. 
Las variables para la construcción de las tasas de incidencia de infección asociada a dispositivos y el porcentaje de uso de dispositivos fueron agregadas por cada institución de salud y notificadas de forma mensual a través de la herramienta en línea.

\section{Vigilancia de la resistencia bacteriana}

La identificación microbiológica y las pruebas de sensibilidad antimicrobiana se realizaron con el sistema automatizado de cada institución (MicroScan ${ }^{\text {TM }}$, Dade Behring, USA; Vitek ${ }^{\text {TM }}$, bioMerieux, France, y Phoenix ${ }^{\mathrm{TM}}$, Becton Dickinson, USA), y por medio del método de concentración inhibitoria mínima (CIM).

Los microorganismos bajo vigilancia fueron los siguientes: Staphylococcus aureus, Enterococcus faecalis, Enterococcus faecium, Klebsiella pneumoniae, Escherichia coli, Enterobacter cloacae, Acinetobacter baumannii y Pseudomonas aeruginosa.

Los antibióticos probados para Gram negativos y no fermentadores fueron: ampicilina, cefazolina, gentamicina, amoxicilina y ácido clavulánico, ampicilina y sulbactam, piperacilina-tazobactam, cefotaxima, ceftriaxona, ceftazidima, cefepime, ertapenem, imipenem, meropenem, trimetoprim-sulfametoxazol, amikacina, ciprofloxacina y colistina. Para las bacterias Gram positivas se evaluaron los siguientes antibióticos: eritromicina, oxacilina, trimetoprim sulfametoxazol, linezolid, tetraciclina, vancomicina, rifampicina, ciprofloxacina, gentamicina y gentamicina de alta carga.

La información obtenida de los equipos automatizados se consolidó en bases de datos en formato Whonet (22) e incluyó las variables de fecha de toma de muestra, tipo de localización, tipo de muestra, microorganismos y las CIM de cada antibiótico probado, las cuales fueron remitidas por las entidades territoriales de salud a través de correo electrónico al Instituto Nacional de Salud, en donde se revisó la calidad de la información. No se incluyeron datos de una de las instituciones debido a que incumplió con el proceso de estandarización establecido por el Instituto Nacional de Salud.

\section{Vigilancia del consumo de antibióticos}

El control del consumo de antibióticos se efectuó siguiendo el sistema de clasificación químicoterapéutica y anatómica con dosis diaria definida (The Anatomical Therapeutic Chemical Classification System with Defined Daily Doses - ATC/DDD) de la Organización Mundial de la Salud (OMS) (23), utilizando como unidad técnica de medida la dosis diaria definida por 100 camas-día (entendida como la dosis diaria promedio de mantenimiento de un medicamento que se use para su indicación principal en adultos), de los siguientes antibióticos parenterales: ceftriaxona, ciprofloxacina, imipenem, meropenem, piperacilina-tazobactam y vancomicina. La clasificación se hizo con base en la información del servicio farmacéutico de cada institución de salud. La información se notificó mensualmente a través de la herramienta de notificación en línea. Sólo se realizó la vigilancia de ciprofloxacina en servicios de hospitalización de adultos diferentes a las unidades de cuidados intensivos.

El flujo de la notificación para todos los eventos siguió los lineamientos establecidos en el Sistema Nacional de Vigilancia en Salud Pública - Sivigila (24).

\section{Análisis de la información}

La información notificada de las infecciones asociadas a dispositivos y de consumo de antibióticos se exportó en el formato de Excel incorporado en la herramienta en línea. Para las infecciones asociadas a dispositivos se estimaron las tasas de incidencia por 1.000 días dispositivo en cada tipo de infección (neumonía asociada a respiración mecánica asistida, infección del torrente sanguíneo asociada a catéter central y de infección sintomática del tracto urinario asociada a catéter urinario) y los porcentajes de utilización de dispositivos por tipo de dispositivo (ventilador, catéter central, catéter urinario) y por tipo de unidad de cuidados intensivos (adulto, pediátrica o neonatal), aplicando las siguientes fórmulas:

Número de casos nuevos de infecciones asociadas a dispositivos en la unidad de cuidados intensivos

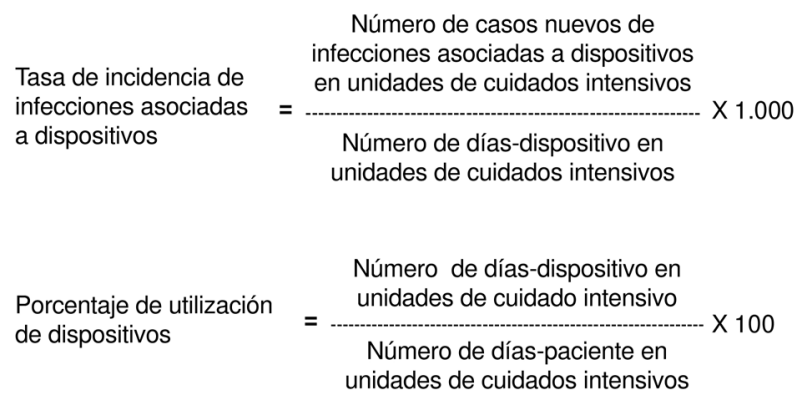

La información de resistencia bacteriana se analizó utilizando el software Whonet 5.6 e incluyendo solo el primer aislamiento de cada paciente discriminado según su permanencia o no en la unidad de cuidados intensivos. La interpretación de los resultados de resistencia se hizo de acuerdo con las recomendaciones del Clinical and Laboratory 
Standards Institute (CLSI) para 2012 (25), excepto para las cefalosporinas de tercera generación y el ertapenem, con los cuales se utilizó la norma del CLSI del 2009 (26).

En cada institución de salud, los consumos de antibióticos se calcularon discriminando por permanencia o no en las unidades de cuidados intensivos de adultos y según la siguiente fórmula:

Dosis diaria definida $=\frac{\text { Número de dosis diaria definida* }}{-\begin{array}{c}\text { Número de camas } \times \text { proporción de } \\ \text { ocupación } \mathrm{x} \text { tiempo (días del mes) }\end{array}}$

${ }^{*} \begin{gathered}\text { Número de dosis } \\ \text { diaria definida }\end{gathered}$
$\begin{gathered}\text { Dosis diaria definida del medicamento }(g) \\ \text { según OMS }\end{gathered}$

\section{Consideraciones éticas}

Dado que se hizo recolección y análisis de información colectiva y que los resultados pretenden generar acciones que aporten a la prevención y control de infecciones, el estudio se clasificó como una investigación sin riesgo y no se requirió consentimiento informado de acuerdo con las normas científicas, técnicas y administrativas para la investigación en salud del Ministerio de Salud de la República de Colombia (27). El manejo de los datos se ajustó a estrictos parámetros de confidencialidad de acuerdo a las leyes vigentes.

\section{Resultados}

\section{Vigilancia de las infecciones asociadas a dispositivos}

Durante el período de notificación se recolectó la información de 34 unidades de cuidados intensivos, de las cuales, 61,8\% correspondió a servicios de las unidades de cuidados intensivos de adultos, $23,5 \%$ a las unidades pediátricas de cuidados intensivos y $14,7 \%$ a las unidades neonatales de cuidados intensivos. Se notificaron 523 casos de infecciones asociadas a dispositivos, 107.969 díasdispositivo y 89.195 días-paciente en unidades de cuidados intensivos. La distribución de las tasas de incidencia de estas infecciones y los porcentajes de utilización de dispositivos a nivel departamental y nacional se presentan en los cuadros 1 y 2 .

En el análisis comparativo entre entes territoriales se observó una amplia variación entre las tasas de infección asociada a dispositivos y los porcentajes de uso de dispositivos. En Valle y Bogotá las tasas

Cuadro 1. Tasas de infección asociada a dispositivos y porcentaje de utilización de dispositivos por ente territorial, Colombia, 2011

\begin{tabular}{|c|c|c|c|c|c|c|c|}
\hline $\begin{array}{l}\text { Tipo de } \\
\text { infección }\end{array}$ & Ente territorial & $\begin{array}{c}\text { Tipo y } \\
\mathbf{N}^{\circ} \mathrm{UCl}\end{array}$ & $\begin{array}{c}\text { Casos } \\
\text { nuevos }\end{array}$ & Días-dispositivo & Días-paciente & Tasa IAD* & $\begin{array}{l}\text { UD } \\
(\%)\end{array}$ \\
\hline \multirow[t]{8}{*}{ ITS-AC } & \multirow[t]{3}{*}{ Antioquia } & Adu (6) & 29 & 8.700 & 12.044 & 3,3 & 72,2 \\
\hline & & Ped (1) & 4 & 734 & 1.221 & 5,4 & 60,1 \\
\hline & & $\mathrm{Neo}(3)$ & 20 & 1.589 & 3.289 & 12,6 & 48,3 \\
\hline & \multirow[t]{3}{*}{ Bogotá } & Adu (3) & 20 & 5.092 & 6.378 & 3,9 & 79,8 \\
\hline & & Ped (1) & 3 & 298 & 522 & 10,1 & 57,1 \\
\hline & & $\mathrm{Neo}(2)$ & 5 & 2.134 & 5.537 & 2,3 & 38,5 \\
\hline & \multirow[t]{2}{*}{ Valle del Cauca } & Adu12) & 95 & 12.422 & 29.385 & 7,6 & 42,3 \\
\hline & & Ped (3) & 33 & 5.045 & 8.546 & 6,5 & 59,0 \\
\hline \multirow[t]{8}{*}{ NAV } & \multirow[t]{3}{*}{ Antioquia } & $\mathrm{Neo}(3)$ & 30 & 7.501 & 22.273 & 4,0 & 33,7 \\
\hline & & Adu (6) & 39 & 7.651 & 12.044 & 5,1 & 63,5 \\
\hline & & Ped (1) & 2 & 550 & 1.221 & 3,6 & 45,0 \\
\hline & \multirow[t]{3}{*}{ Bogotá } & $\mathrm{Neo}(3)$ & 3 & 619 & 3.289 & 4,8 & 18,8 \\
\hline & & Adu (3) & 11 & 3.577 & 6.378 & 3,1 & 56,1 \\
\hline & & Ped (1) & 1 & 127 & 522 & 7,9 & 24,3 \\
\hline & \multirow[t]{2}{*}{ Valle del Cauca } & $\mathrm{Neo}(2)$ & 1 & 671 & 5.537 & 1,5 & 12,1 \\
\hline & & Adu (1) & 49 & 10.578 & 29.385 & 4,6 & 36,0 \\
\hline \multirow[t]{8}{*}{ ISTU-AC } & \multirow[t]{3}{*}{ Antioquia } & Ped (3) & 16 & 2788 & 8.546 & 5,7 & 32,6 \\
\hline & & Neo (3) & 11 & 3.082 & 22.273 & 3,6 & 13,8 \\
\hline & & Adu (6) & 60 & 9.534 & 12.044 & 6,3 & 79,2 \\
\hline & \multirow[t]{3}{*}{ Bogotá } & Ped (1) & 2 & 511 & 1.221 & 3,9 & 41,9 \\
\hline & & Adu (3) & 16 & 5.340 & 6.378 & 3,0 & 83,7 \\
\hline & & Ped (1) & 0 & 93 & 522 & 0,0 & 17,8 \\
\hline & \multirow[t]{2}{*}{ Valle del Cauca } & Adu (12) & 68 & 16.795 & 29.385 & 4,0 & 57,2 \\
\hline & & Ped (3) & 5 & 2.538 & 8.546 & 2,0 & 29,7 \\
\hline
\end{tabular}

UCI: unidad de cuidados intensivos; IAD: infecciones asociadas a dispositivos; UD: utilización de dispositivos invasivos

* Tasa de infección asociada a dispositivo por 1.000 días-dispositivo

ITS-AC: infección del torrente sanguíneo asociada a catéter; NAV: neumonía asociada a respirador mecánico; ISTU-AC: infección sintomática del tracto urinario asociada a catéter

Adu: adulto; Ped: pediátrica; Neo: neonatal. 

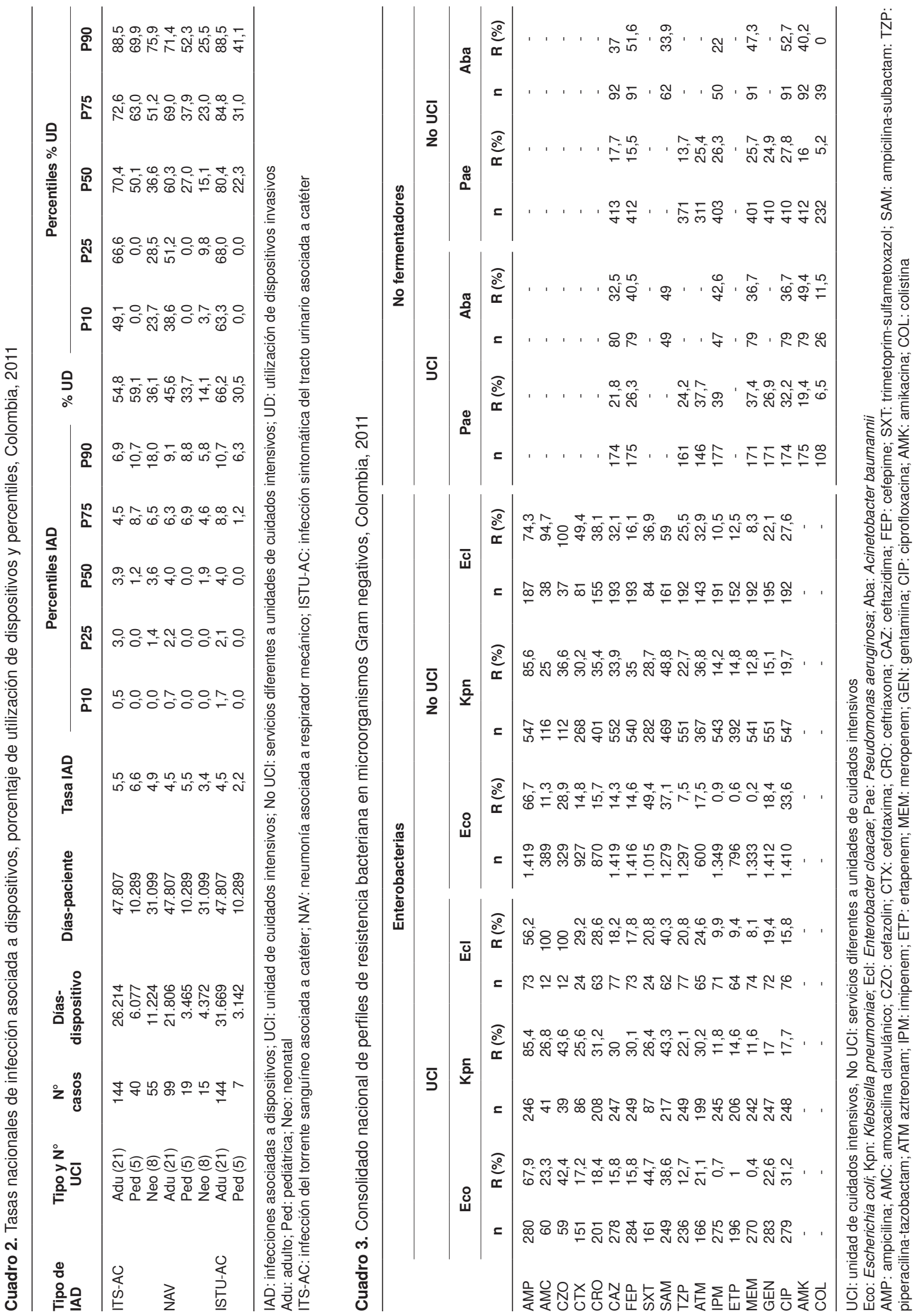
más altas de infección asociada a dispositivos se encontraron en las infecciones del torrente sanguíneo asociadas a catéter central en los tres tipos de unidad de cuidados intensivos; una situación diferente se presentó en Antioquia, donde en las unidades de cuidados intensivos de adultos predominaron las infecciones sintomáticas del tracto urinario asociadas a catéter urinario y en las unidades de cuidados intensivos pediátricas y neonatales, las infecciones del torrente sanguíneo asociadas a catéter central.

Del total de estas infecciones en unidad de cuidados intensivos reportadas a nivel nacional, las infecciones del torrente sanguíneo asociadas a catéter central representaron $45,7 \%$, las infecciones sintomáticas del tracto urinario asociadas a catéter urinario, 28,9 \%, y las neumonías asociadas al respirador, 25,4\%.

\section{Vigilancia de resistencia bacteriana}

Se analizaron 8.330 aislamientos, de los cuales, $25,4 \%$ provenía de las unidades de cuidados intensivos y $74,6 \%$, de otras áreas de hospitalización. En las figuras 1 y 2 se presentan las frecuencias de los microorganismos y los tipos de muestra discriminadas por tipo de localización (unidad de cuidados intensivos y otras áreas).

De 590 aislamientos obtenidos en muestras de sangre en las unidades de cuidados intensivos, $12 \%$ correspondió a K. pneumoniae, $10,7 \%$ a $S$. aureus y $5,4 \%$ a E. coli; de los 1.010 aislamientos para este mismo tipo de muestra en servicios de otras áreas de hospitalización, 13,4 \% correspondió a $S$. aureus, $12,9 \%$ a $E$. coli y $8,7 \%$ a $K$. pneumoniae. En muestras de orina se aislaron 405 microorganismos en las unidades de cuidados intensivos, de los cuales, 33,1 \% correspondió a

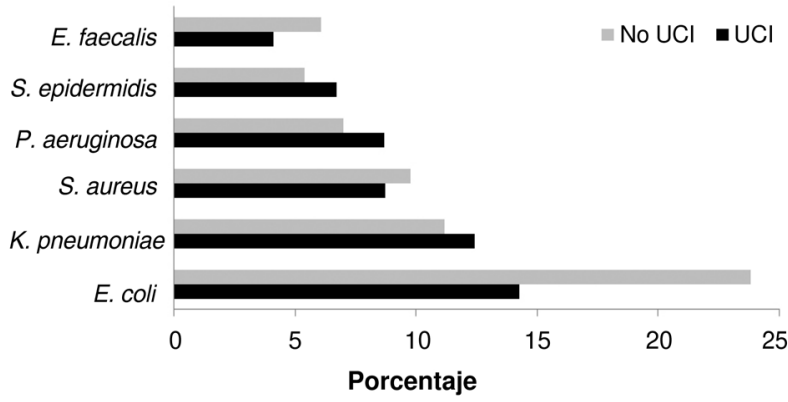

UCI: unidad de cuidados intensivos

No UCI: servicios de hospitalización diferentes a la UCI

Figura 1. Frecuencia de microorganismos por tipo de localización, Colombia, 2011

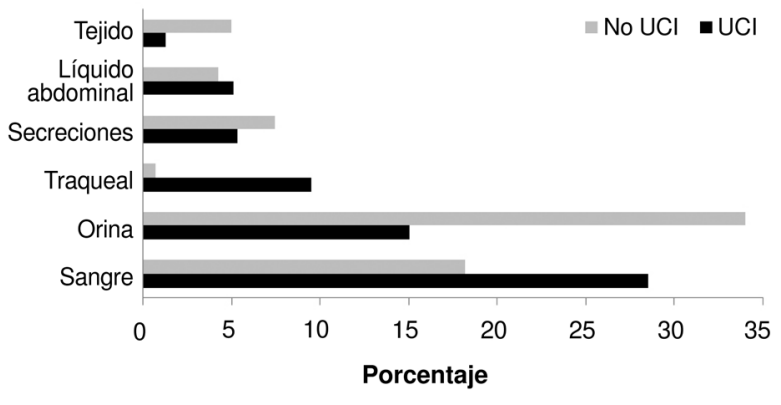

UCI: unidad de cuidados intensivos

No UCI: servicios de hospitalización diferentes a la UCI

Figura 2. Frecuencia de muestras por tipo de localización, Colombia, 2011

E. coli y $15,3 \%$ a K. pneumoniae, mientras que en otros servicios de hospitalización se identificaron 1.990 asilamientos, de los cuales, $4 \%$ fue de $E$. coli y $11 \%$ de K. pneumoniae.

\section{Perfiles de resistencia en microorganismos Gram negativos}

En los cuadros 3 y 4 se pueden observar los perfiles de resistencia en los microorganismos Gram negativos y bacterias no fermentadoras discriminados por tipo de localización (unidades de cuidados intensivos y otros servicios de hospitalización).

En el análisis de muestras de sangre, el perfil de resistencia en las unidades de cuidados intensivos mostró para E. coli un elevado porcentaje de resistencia a cefotaxima (37,5\%), en tanto que en orina se observó resistencia a ceftriaxona $(19,2 \%)$, mientras que K. pneumoniae en muestras de sangre mostró porcentajes de resistencia similares para ceftazidima y ceftriaxona $(36,6 \%)$ y en muestras de orina se observó un porcentaje de resistencia elevado para ceftriaxona (44,3\%). En otros servicios hospitalarios diferentes a las unidades de cuidados intensivos, la resistencia en aislamientos de $E$. coli en muestras de sangre se presentó principalmente en ceftriaxona con $10,7 \%$ y en orina con $15 \%$, mientras que en $K$. pneumoniae en muestras de sangre se observó una mayor resistencia frente a cefalosporinas de tercera generación que superó el 29,8 \% y en orina, el 34,4\%.

En las unidades de cuidados intensivos se destacó para $K$. pneumoniae en muestras de sangre una elevada resistencia a carbapenémicos, específicamente al ertapenem, de $15,1 \%$, y en otros servicios diferentes a las unidades de cuidados intensivos, de alrededor de $10 \%$. 
Cuadro 4. Consolidado nacional de perfiles de resistencia bacteriana de microorganismos Gram positivos, Colombia, 2011

\begin{tabular}{|c|c|c|c|c|c|c|c|c|c|c|}
\hline & \multicolumn{10}{|c|}{ Gram positivos } \\
\hline & \multicolumn{4}{|c|}{$\mathrm{UCl}$} & \multicolumn{6}{|c|}{ No UCI } \\
\hline & \multicolumn{2}{|c|}{ Sau } & \multicolumn{2}{|c|}{ Efm } & \multicolumn{2}{|c|}{ Sau } & \multicolumn{2}{|c|}{ Efm } & \multicolumn{2}{|c|}{ Efa } \\
\hline & $\mathbf{n}$ & $\mathbf{R}(\%)$ & $\mathbf{n}$ & $\mathbf{R}(\%)$ & $\mathbf{n}$ & $\mathbf{R}(\%)$ & $\mathbf{n}$ & $\mathbf{R}(\%)$ & $\mathbf{n}$ & $\mathbf{R}(\%$ \\
\hline GEN & 171 & 15,2 & - & - & 659 & 8,8 & - & - & - & - \\
\hline $\mathrm{CIP}$ & 173 & 13,3 & - & - & 662 & 9,8 & - & - & - & - \\
\hline SXT & 180 & 5 & - & - & 667 & 1,2 & - & - & - & - \\
\hline OXA & 181 & 27,6 & - & - & 671 & 34,3 & - & - & - & - \\
\hline RIF & 180 & 1,1 & - & - & 669 & 1,2 & - & - & - & - \\
\hline CLI & 178 & 17,4 & - & - & 670 & 11 & - & - & - & - \\
\hline ERY & 178 & 27 & - & - & 669 & 20,9 & - & - & - & - \\
\hline TCY & 172 & 20,9 & - & - & 657 & 23,3 & - & - & - & - \\
\hline LNZ & 180 & 0,6 & 23 & 0 & 658 & 0,5 & 54 & 0 & 335 & 0,9 \\
\hline VAN & 179 & 0 & 23 & 34,8 & 667 & 0 & 54 & 27,8 & 334 & 0 \\
\hline GEH & - & - & 23 & 30,4 & - & - & 53 & 22,6 & 318 & 19,2 \\
\hline AMP & - & - & 23 & 95,7 & - & - & 56 & 73,2 & 324 & 1,2 \\
\hline
\end{tabular}

UCI: unidad de cuidados intensivos, No UCl: servicios diferentes a unidades de cuidados intensivos

Sau: Staphylococcus aureus; Efm: Enterococo faecium; Efa: Enterococo faecalis

GEN: gentamicina; CIP: ciprofloxacina; SXT: trimetoprim-sulfametoxazol; OXA: oxacilina; RIF: rifampicina; CLI: clindamicina; ERY: eritromicina; TCY:

tetraciclina; LNZ: linezolid; VAN: vancomicina; GEH: gentamicina de alta carga; AMP: ampicilina

\section{Perfiles de resistencia en microorganismos Gram positivos}

En el cuadro 4 se presenta el perfil de resistencia en microorganismos Gram positivos en las unidades de cuidados intensivos y en otros servicios diferentes a estas. En las unidades de cuidados intensivos no se reportaron aislamientos de $E$. faecalis. Ningún aislamiento de $S$. aureus presentó sensibilidad disminuida a vancomicina.

\section{Tendencias de los fenotipos de resistencia bacteriana por ente territorial}

Se observó que el departamento de Valle del Cauca presentaba los porcentajes más altos del fenotipo de $E$. coli resistente a cefalosporinas de tercera generación tanto en unidades de cuidados intensivos como en otros servicios, fundamentalmente a cefotaxima y ceftriaxona. En los departamentos de Valle y Antioquia el comportamiento de $K$. pneumoniae resistente a cefalosporinas de tercera generación en servicios diferentes a las unidades de cuidados intensivos fue superior a $30 \%$, mientras que en Bogotá la resistencia a cefalosporinas de tercera generación en las unidades de cuidados intensivos superó el $20 \%$. Enterobacter cloacae presentó mayores porcentajes de resistencia a cefalosporinas de tercera generación en los tres departamentos, siendo dichos porcentajes mayores en otros servicios diferentes a las unidades de cuidados intensivos.
Antioquia presentó los mayores porcentajes de resistencia en K. pneumoniae, siendo el ertapenem el marcador más sensible en los servicios diferentes a las unidades de cuidados intensivos $(24,4 \%)$.

El comportamiento de los principales fenotipos de resistencia en Gram negativos no fermentadores mostró que a pesar del bajo número de aislamientos de $A$. baumannii en Bogotá, todos los aislamientos en servicios diferentes a las unidades de cuidados intensivos fueron resistentes a meropenem, en tanto que en las unidades de cuidados intensivos, $25 \%$ de ellos fue resistente a imipenem; en Antioquia se presentaron los mayores porcentajes de resistencia en las unidades de cuidados intensivos, principalmente a imipenem (80\%) y en el Valle, a meropenem (57,1\%) en servicios diferentes de las unidades de cuidados intensivos (figuras 3 y 4).

Con respecto a $S$. aureus resistente a meticilina, el Valle presentó la mayor resistencia en las unidades de cuidados intensivos (43,9\%), en tanto que en Antioquia se presentó mayor proporción de $E$. faecium resistente a vancomicina en servicios diferentes a las unidades de cuidados intensivos (71,4 \%) (figura 5).

\section{Resultados de la vigilancia del consumo de antibióticos}

Se contó con información de 211 camas de las unidades de cuidados intensivos y 2.424 camas de los servicios de hospitalización de adultos 


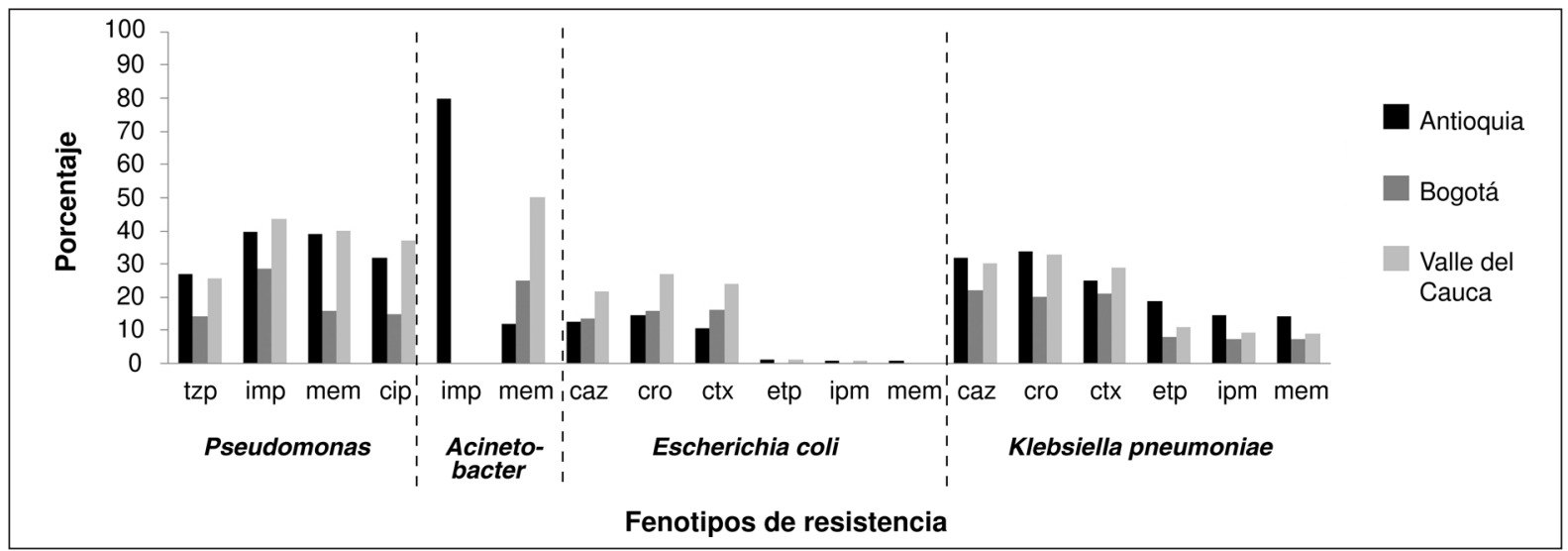

Tzp: piperacilina-tazobactam; imp: imipenem; mem: meropenem; cip: ciprofloxacina; caz: ceftazidime; cro: ceftriaxona; ctx: cefotaxima; etp: ertapenem

Figura 3. Fenotipos de resistencia bacteriana en microorganismos Gram negativos en unidades de cuidados intensivos, Colombia, 2011

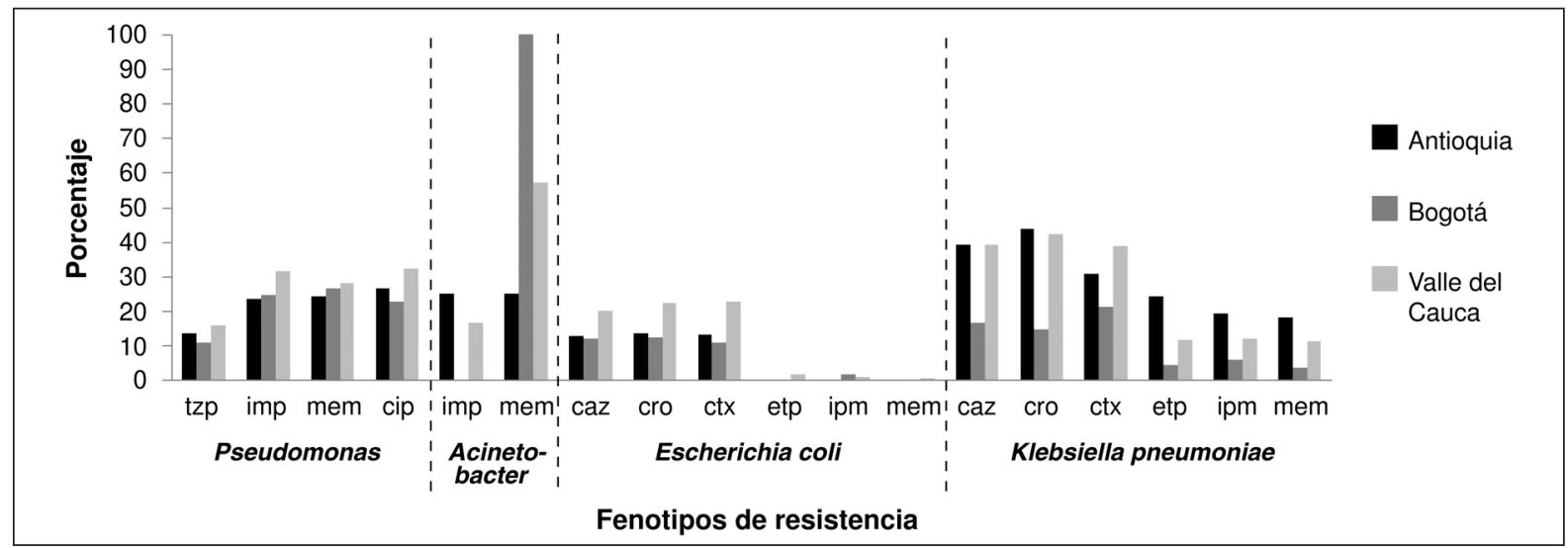

Tzp: piperacilina-tazobactam; imp: imipenem; mem: meropenem; cip: ciprofloxacina; caz: ceftazidime; cro: ceftriaxona; ctx: cefotaxima; etp: ertapenem

Figura 4. Fenotipos de resistencia bacteriana en microorganismos Gram negativos en servicios diferentes a las unidades de cuidados intensivos, Colombia, 2011

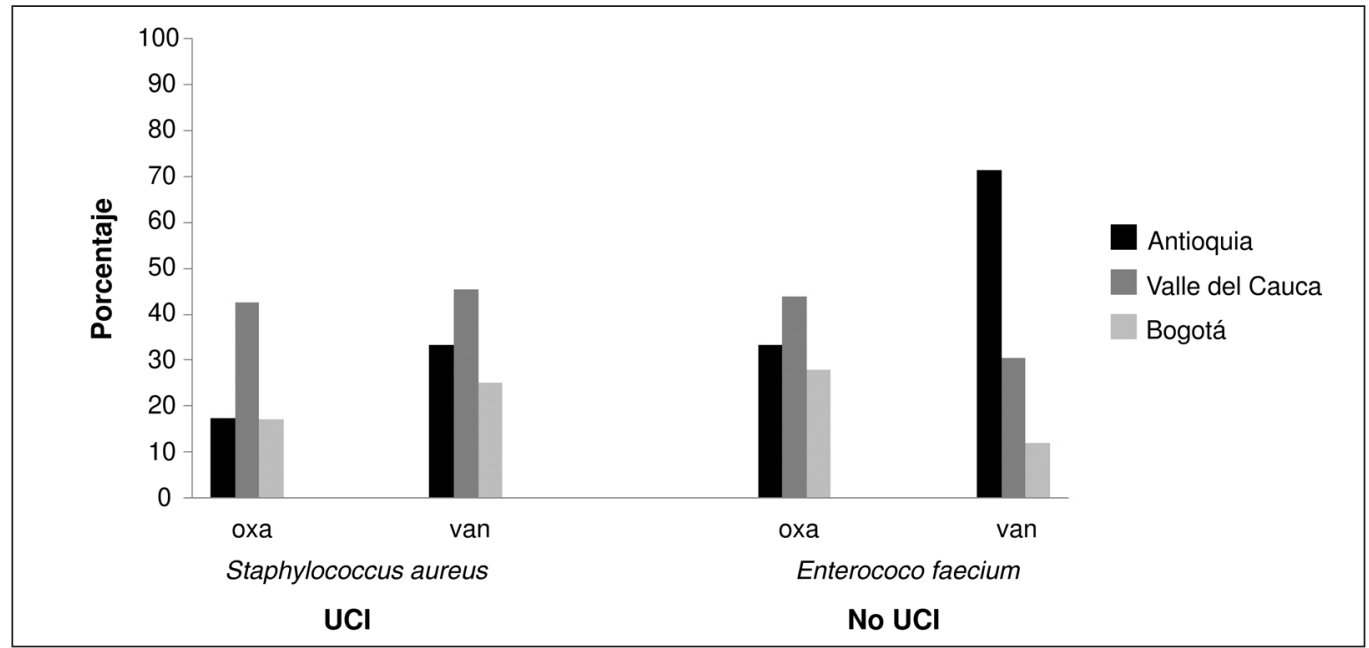

oxa: oxacilina; van: vancomicina. UCl: unidad de cuidados intensivos. No UCl: servicios diferentes a unidades de cuidados intensivos.

Figura 5. Fenotipos de resistencia bacteriana en microorganismos Gram positivos en unidades de cuidados intensivos y en áreas diferentes a las unidades de cuidado intensivo, Colombia, 2011 
Cuadro 5. Consolidado nacional de dosis diarias definidas de antibióticos de uso parenteral por tipo de localización, Colombia, 2011

\begin{tabular}{|c|c|c|c|c|c|c|c|c|c|c|c|c|c|}
\hline & \multirow{2}{*}{\multicolumn{2}{|c|}{ No. de camas }} & \multicolumn{11}{|c|}{ Dosis diarias definidas $x 100$ camas día } \\
\hline & & & \multicolumn{2}{|c|}{ Ceftriaxona } & \multirow{2}{*}{$\frac{\text { Ciprofloxacina }}{\text { No UCl }}$} & \multicolumn{2}{|c|}{ Imipenem } & \multicolumn{2}{|c|}{ Meropenem } & \multicolumn{2}{|c|}{$\begin{array}{l}\text { Piperacilina- } \\
\text { tazobactam }\end{array}$} & \multicolumn{2}{|c|}{ Vancomicina } \\
\hline & $\mathrm{UCl}$ & No UCl & $\mathrm{UCl}$ & No UCI & & $\mathrm{UCl}$ & $\begin{array}{l}\text { No } \\
\text { UCI }\end{array}$ & UCl & No UCl & $\mathrm{UCl}$ & $\begin{array}{l}\text { No } \\
\text { UCI }\end{array}$ & $\mathrm{UCl}$ & No UCl \\
\hline Antioquia & 62 & 1.113 & 1,59 & 1,6 & 7,0 & 1,08 & 0,4 & 30,13 & 5,3 & 8,65 & 8,9 & 13,47 & 3,6 \\
\hline Bogotá & 27 & 381 & 2,67 & 4,6 & 2,2 & 0,02 & 0,1 & 13,69 & 5,2 & 10,5 & 7,3 & 6,74 & 3,3 \\
\hline Valle & 122 & 930 & 7,77 & 3,7 & 2,7 & 0,04 & 0,0 & 20,99 & 6,0 & 10,32 & 4,2 & 14,25 & 4,8 \\
\hline Nacional & 211 & 2.424 & 5,37 & 2,8 & 4,6 & 0,33 & 0,2 & 22,45 & 5,5 & 9,72 & 6,8 & 13 & 3,9 \\
\hline
\end{tabular}

UCI: unidad de cuidados intensivos, No UCl: servicios diferentes a unidades de cuidados intensivos.

diferentes a las unidades de cuidados intensivos. Los resultados de las dosis diarias definidas por 100 camas día se encuentran en el cuadro 5.

La frecuencia del consumo de los antibióticos bajo vigilancia fue, en general, mayor en los servicios de cuidados intensivos que en el resto de los servicios de hospitalización de adultos. En las instituciones de salud de las tres entidades territoriales se presentó una mayor frecuencia de consumo de meropenem en las unidades de cuidados intensivos, seguido de vancomicina en Antioquia y Valle del Cauca, y piperacilina-tazobactam en Bogotá y Valle del Cauca. En servicios diferentes a las unidades de cuidados intensivos la mayor frecuencia se observó con piperacilina-tazobactam en instituciones de Antioquia y Bogotá, seguido de ciprofloxacina en Antioquia y meropenem en las tres entidades territoriales.

\section{Discusión}

El presente artículo presenta los primeros resultados de la implementación de las estrategias de vigilancia de infecciones asociadas a dispositivos, resistencia y consumo de antibióticos puestas a prueba en 10 instituciones de salud de alta complejidad del país.

Todas las instituciones de salud en Colombia, en especial las de media y alta complejidad, cuentan con equipos multidisciplinarios (comités de infecciones) que apoyan las labores de vigilancia, prevención y control de infecciones asociadas a la atención en salud, cuya organización y funcionamiento es definida por cada una de ellas de manera independiente. Las características de la población atendida por las instituciones varía de acuerdo con el tipo de aseguramiento, el grado de especialidad de los servicios ofrecidos y los recursos de atención, tales como el talento humano y el acceso a la tecnología, entre otros.
Asimismo, el grado de desarrollo de los programas de prevención y control de infecciones asociadas a la atención en salud es heterogéneo, e incluye desde instituciones que no tienen tal tipo de programa hasta aquellas que lo tienen en un nivel muy avanzado. Las instituciones participantes en la prueba piloto reunían estas características, lo cual permitió probar las herramientas de vigilancia en escenarios distintos, reconociendo desde un comienzo que esto podría limitar la posibilidad de comparar los indicadores reportados durante la fase de implementación de la prueba.

Por otro lado, el conocimiento de la problemática de estas infecciones y la resistencia bacteriana en nuestro medio se considera sectorizado y ligado a las investigaciones de grupos de trabajo independientes ubicados en las regiones más pobladas del país, lo cual dificulta una aproximación real a la situación nacional. Por esta razón, el diseño de las estrategias de vigilancia puestas a prueba consideró la experiencia de las iniciativas locales (18), así como las recomendaciones definidas para el desarrollo de sistemas de vigilancia en salud pública de las infecciones asociadas a la atención en salud y a la resistencia bacteriana que han resultado exitosos en otros países (13,14,2830), sin perder de vista la heterogeneidad de las instituciones del país.

En cuanto a las tasas nacionales de infecciones asociadas a dispositivos, se observó que todas se encuentran por debajo de lo reportado por el estudio INICC, llevado a cabo en varios países del mundo de escasos recursos (31), pero mayores a las reportadas por el NHSN/CDC de los Estados Unidos (32); esto último era de esperarse debido al número limitado de instituciones participantes y las características propias del sistema de salud colombiano, que dificultan las comparaciones con otros sistemas de salud. Es por esto que la 
información aquí presentada es solo un indicador general y debe interpretarse teniendo en cuenta las características de cada institución y la región del país, ya que muestran un comportamiento diferente (cuadro 1).

La experiencia de la vigilancia de infecciones asociadas a la atención en salud en las unidades neonatales de cuidados intensivos basada en la categoría de peso al nacer, como lo recomiendan algunos autores, no se consideró entre los objetivos de la prueba piloto (13); sin embargo, el estudio permitió un primer acercamiento al conocimiento de la dinámica de la vigilancia en estos servicios que sugiere la necesidad de mediciones ajustadas al tipo de riesgo en esta población más vulnerable.

La preocupación por el aumento de microorganismos resistentes se justifica a pesar de las limitaciones de la prueba piloto. Tal es el caso de las enterobacterias, pues se confirmó la elevada resistencia a las cefalosporinas de tercera generación, lo que sugiere la presencia de betalactamasas de espectro extendido (BLEE), como lo han reportado varios autores (17,33-35). La resistencia a ciprofloxacina en $E$. coli $(31,2 \%$ en unidades de cuidados intensivos y $33,6 \%$ en otros servicios diferentes a las unidades de cuidados intensivos) supera lo reportado por el distrito capital $(27,9 \%$ en unidades de cuidados intensivos y $23,3 \%$ en otros servicios) y por algunos grupos de investigación del país (3639), lo que indica una posible asociación entre la producción de BLEE y la resistencia a quinolonas y aminoglucósidos.

La diseminación de carbapenemasas en bacterias Gram negativas y no fermentadoras es un problema de salud pública debido a las pocas opciones terapéuticas que actualmente existen en el mercado para tratar pacientes afectados por estos microorganismos, evidenciándose un incremento en la prevalencia de este mecanismo de resistencia en Colombia durante los últimos años, especialmente en $K$. pneumoniae (4042). Ertapenem se destaca como el marcador más sensible, con una resistencia superior a $14 \%$, muy similar a la de Bogotá (13\%). Sin embargo, al comparar estos datos con los reportados por cada ente territorial, Antioquia se caracterizó por presentar una elevada resistencia a carbapenémicos, que alcanza el $24,4 \%$ en los servicios de hospitalización, mientras que en $E$. coli, los porcentajes de resistencia fueron bajos.
Nuestro ámbito hospitalario se ha caracterizado por presentar un comportamiento de multirresistencia en los aislamientos de $A$. baumannii, principalmente en las unidades de cuidados intensivos (43), lo que se demostró en el presente estudio. Sin embargo, los datos muestran, en general, una disminución en la resistencia a carbapanémicos y cefalosporinas en comparación con datos publicados anteriormente $(35,37,44)$. En el caso de los aislamientos de $P$. aeruginosa, se mantuvo el perfil de multirresistencia, observándose un incremento en la resistencia a carbapenémicos en las unidades de cuidados intensivos, aunque menor que lo reportado por otros autores $(35,37,44)$.

Por otra parte, los datos encontrados para $S$. aureus sugieren un perfil de alta resistencia a oxacilina comparada con otros antimicrobianos como eritromicina, clindamicina, rifampicina y ciprofloxacina y compatible con el perfil comunitario, lo cual es similar a lo reportado por otros estudios $(36,38)$.

Aunque el número de aislamientos de $E$. faecium era bajo, se destacó su elevada resistencia a vancomicina, principalmente en las unidades de cuidados intensivos, lo que constituye un problema de salud pública debido a la dificultad del tratamiento y a la fácil diseminación de este mecanismo de resistencia a otras especies bacterianas.

Estos tipos de resistencia confirman una vez más la necesidad de fortalecer las acciones de prevención y control para evitar su diseminación en la población, incluido un mejor uso de los antimicrobianos. En este sentido, la vigilancia del consumo de antibióticos se articula como un componente transversal que contribuye de manera indirecta al conocimiento de las prácticas de prescripción de antibióticos y de la expresión de resistencia bacteriana y que podría, por ende, orientar las acciones hacia un uso más racional de estos medicamentos en las instituciones de salud del país (45).

Si bien existen instituciones que cuentan con mediciones del consumo de antibióticos, no siempre su metodología de medición es la misma y son pocas las publicaciones con relación a este tema en el país $(46,47)$. El uso de antibióticos es el principal factor asociado con la aparición de resistencia, sin embargo, evaluar esta relación representa una gran complejidad, dado que las interacciones entre el uso de antibióticos y la resistencia no son necesariamente directas, lo que significa que el consumo de un antibiótico puede afectar microorganismos que no son su 
blanco terapéutico e inducir resistencias como la que se ha documentado en el caso del uso de la ciprofloxacina y la aparición de $S$. aureus resistente a meticilina (48). Este, y otro tipo de análisis, implica disponer de datos que provienen principalmente de sistemas de vigilancia tanto de la resistencia bacteriana como del consumo de antibióticos. Sin embargo, el acceso a esta información exige la estandarización de una metodología de medición en las instituciones de salud $(22,49,50)$.

En la información recolectada durante la prueba piloto llama la atención el consumo de carbapenémicos debido a su amplio espectro de actividad y el impacto potencial de su uso excesivo en la aparición de resistencia bacteriana. Es probable que los datos de consumo de meropenem observados en el presente estudio se relacionen con una mayor proporción de resistencia a este medicamento en enterobacterias, especialmente en $K$. pneumoniae, sin embargo, se requieren mayores estudios que aporten al conocimiento de esta problemática en el país.

Los datos aquí presentados reflejan la necesidad de implementar y estandarizar la vigilancia a nivel nacional y aportan una experiencia importante para el mejoramiento de las herramientas puestas a prueba para la vigilancia de estos eventos, lo cual permitirá fortalecer el Sistema Nacional de Vigilancia en Salud Pública - Sivigila (24), la detección en el laboratorio de microorganismos emergentes de importancia hospitalaria, y la adopción de acciones gubernamentales e institucionales para la prevención y control de infecciones, la contención de la resistencia y la promoción del uso prudente de antibióticos, acompañadas de un proceso de seguimiento y supervisión.

\section{Agradecimientos}

Agradecemos a las instituciones de salud participantes, a las entidades territoriales de salud de Antioquia, Medellín, Valle del Cauca, Cali y Bogotá y a la Organización Panamericana de la Salud.

\section{Conflicto de intereses}

Los autores declaran no tener conflictos de intereses en la realización del presente estudio.

\section{Financiación}

Este estudio fue financiado por el Ministerio de Salud y la Protección Social y el Instituto Nacional de Salud de Colombia por medio del Convenio Interadministrativo 081 de 2010.

\section{Referencias}

1. Klevens RM, Edwards JR, Richards CL, Horan T, Gaynes R, Pollock D, et al. Estimating healthcare-associated infections in U.S. hospitals, 2002. Public Health Rep. 2007;122:160-6.

2. Gudiol F, Limón E, Fondevilla E, Argimon J, Almirante B, Pujol M. The development and successful implementation of the VINCat Program. Enferm Infecc Microbiol Clin. 2012;30(Supl.3):3-6. http://dx.doi.org/10.1016/S0213-005X (12)70089-7

3. Haley RW, Culver DH, White JW, Morgan WM, Emori TG, Munn VP, et al. The efficacy on infection surveillance and control programs in preventing nosocomial infections in US hospitals. Am J Epidemiol. 1985;121:182-205.

4. Organización Panamericana de la Salud. Una atención más limpia es una atención más segura. Fecha de consulta: 16 de agosto de 2013. Disponible en: http://www.who.int/ gpsc/background/es/index.html.

5. World Health Organization. Report on the Burden of Endemic Health Care-Associated Infection Worldwide 2011. Fecha de consulta: 16 de agosto de 2013. Disponible en: http://apps.who.int/iris/bitstream/10665/80135/1/97892 41501507_eng.pdf.

6. U.S. Department of Health and Human Services. National action plan to prevent healthcare-associated infections: Road map to elimination - 2013. Fecha de consulta: 9 de octubre de 2013. Disponible en: http://www.hhs.gov/ash/initiatives/ hai/actionplan/hai-action-plan-executive-summary.pdf.

7. World Health Organization. WHO global strategy for containment of antimicrobial resistance - 2001. Fecha de consulta: 27 de mayo de 2013. Disponible en: http://www. antibioticos.msc.es/PDF/resist_OMS_estrategia_mundial_ resumen.pdf.

8. Shlaes DM, Gerding DN, John JF Jr, Craig WA, Bornstein DL, Duncan RA, et al. Society for Healthcare Epidemiology of America and Infectious Diseases Society of America Joint Committee on the Prevention of Antimicrobial Resistance: Guidelines for the prevention of antimicrobial resistance in hospitals. Infect Control Hosp Epidemiol. 1997;18:275-91.

9. Cosgrove S. The relationship between antimicrobial resistance and patient outcomes: Mortality, length of hospital stay, and health care costs. Clin Infect Dis 2006;42(Supl.2): S82-9. http://dx.doi.org/10.1086/499406

10. Ministerio de Salud de la Nación de Argentina. Programa Nacional de Epidemiología y Control de Infecciones Hospitalarias (VIHDA). Fecha de consulta: 28 de mayo de 2013. Disponible en: http://www.vihda.gov.ar/.

11. Ministerio de Salud de Chile. Infecciones Intrahospitalarias y asociadas a la atención en Salud. Fecha de consulta: 28 de mayo de 2013. Disponible en: http://www.minsal.gob. $\mathrm{cl} / \mathrm{portal} / \mathrm{url} / \mathrm{page} / \mathrm{minsalcl} / \mathrm{g}$ _problemas/g_infecciones intrahospitalarias/infeccionesintra_home.html.

12. Ministerio de Salud Pública de Uruguay. Control de infecciones intrahospitalarias. Fecha de consulta: 28 de mayo de 2013. Disponible en: http://www.msp.gub.uy/ categoriaepidemiologia_138_1_1.html.

13. Centers for Disease Control and Prevention. National Healthcare Safety Network. Fecha de consulta: 28 de mayo de 2013. Disponible en: http://www.cdc.gov/nhsn/faqs/FAQ general.html. 
14. Public Health Agency of Canada. The Canadian Nosocomial Infection Surveillance Program. Fecha de consulta: 29 de mayo de 2013. Disponible en: http://www.phac-aspc.gc.ca/ nois-sinp/survprog-eng.php.

15. Agencia de los Estados Unidos para el Desarrollo Internacional, USAID, Organización Panamericana de la Salud. Infecciones hospitalarias, legislación en América Latina, 2007. Fecha de consulta: 28 de mayo de 2013. Disponible en: http://www.amro.who.int/Spanish/AD/DPC/CD/ amr-infecchospital-legislal.pdf.

16. Bolis M. Infecciones hospitalarias. Legislación en América Latina. Washington, D.C.: Organización Panamericana de la Salud; 2007

17. Acosta S, Aragón J, Benoit S, Betancourt M, Clara L, Figueiredo S, et al. Evaluación de la infección hospitalaria en siete países latinoamericanos. Rev Panam Infectol. 2008;10:S112-22.

18. Villalobos AP, Díaz MH, Barrero LI, Rivera SA, Henríquez DE, Villegas MV, et al. Tendencias de los fenotipos de resistencia bacteriana en hospitales públicos y privados de alta complejidad de Colombia. Rev Panam Salud Publica 2011;30:627-33. http://dx.doi.org/10.1590/S102049892011001200022

19. Secretaría Distrital de Salud de Bogotá. Vigilancia en Salud Pública. Fecha de consulta: 29 de mayo de 2013. Disponible en: http://www.saludcapital.gov.co/sitios/ VigilanciaSaludPublica/ Paginas/IHH.aspx.

20. Casellas JM. Resistencia a los antibacterianos en América Latina: consecuencias para la infectología. Rev Panam Salud Publica. 2011;30:519-28.

21. Yáñez R, Cuadra R. La técnica Delphi y la investigación en los servicios de salud. Cienc Enferm. 2008;14:9-15.

22. Secretaría Distrital de Salud de Bogotá. Criterios diagnósticos de infecciones asociadas al cuidado de la salud a ser utilizados para la notificación al subsistema de vigilancia epidemiológica de IACS en Bogotá, D.C. Adaptado para Bogotá con base en " $\mathrm{CDC} / \mathrm{NHSN}$ surveillance definition of health care-associated infection and criteria for specific types of infections in the acute care setting"-2010. Fecha de consulta: 28 de mayo de 2013. Disponible en: http:// www.saludcapital.gov.co/sitios/VigilanciaSaludPublica/ SiteCollectionDocuments/Criterios\%20diagnosticos\%20 IACS\%20para\%20Bogot\%C3\%A1.pdf.

23. Whonet. Fecha de consulta: 9 de octubre de 2013 Disponible en: http://www.whonet.org/DNN.

24. World Health Organization. WHO Collaborating Centre for Drug Statistics Methodology, Guidelines for ATC classification and DDD assignment 2012. Oslo, 2011. Fecha de consulta: 28 de mayo de 2013. Disponible en: http:// www.whocc.no/atc_ddd_publications/guidelines/.

25. Ministerio de la Protección Social. Decreto por el cual se crea y reglamenta el Sistema de Vigilancia en Salud Pública y se dictan otras disposiciones. Decreto 3518 de 2006. Bogotá: Ministerio de la Protección Social; 2006.

26. Clinical and Laboratory Standards Institute. Performance standards for antimicrobial susceptibility testing; TwentySecond Informational Supplement. M100-S22. 2012;32:4494. Wayne: CLSI; 2012

27. Clinical and Laboratory Standards Institute. Performance standards for antimicrobial susceptibility testing; Twenty-
Second Informational Supplement. M100-S19. 2009;28:3862. Wayne: CLSI; 2009.

28. Ministerio de Salud de Colombia. Resolución 8430 del 4 de octubre de 1993. Fecha de consulta: 29 de mayo de 2013. Disponible en: http://issuu.com/scpbogota/docs/ resolucion_8430.

29. CDC Guidelines Working Group. Updated guidelines for evaluating public health surveillance systems. MMWR. 2001;50:1-35. Fecha de consulta: 29 de mayo de 2013. Disponible en: http://www.cdc.gov/mmwr/preview/mmwrhtml/ rr5013a1.htm.

30. Tokars Jl, Richards C, Andrus M, Klevens M, Curtis A, Horan $\mathrm{T}$, et al. The changing face of surveillance for health care-associated infections. Clin Infect Dis. 2004;39:134752. http://dx.doi.org/10.1086/425000

31. Gastmeier P, Geffers C, Brandt C, Zuschneid I, Sohr D, Schwab F, et al. Effectiveness of a nationwide nosocomial infection surveillance system for reducing nosocomial infections. J Hosp Infect. 2006;64:16-22. http://dx.doi.org/10. 1016/j.jhin.2006.04.017

32. Rosenthal VD, Bijie H, Maki DG, Mehta Y, Apisarnthanarak A, Medeiros EA, et al. International Nosocomial Infection Control Consortium (INICC) report, data summary of 36 countries, for 2004-2009. Am J Infect Control. 2012;40:396407. http://dx.doi.org/10.1016/j.ajic.2011.05.020

33. Dudeck MA, Horan TC, Peterson KD, Allen-Bridson K, Morrell G, Pollock DA, et al. National Healthcare Safety Network (NHSN) report, data summary for 2010, deviceassociated module. Am J Infect Control. 2011;39:798-816. http://dx.doi.org/10.1016/j.ajic.2011.10.001

34. Villegas M, Correa A, Pérez F, Zuluaga T, Radice M, Gutkind G, et al. CTX-M-12 beta-lactamase in a Klebsiella pneumoniae clinical isolate in Colombia. Antimicrob Agents Chemother. 2004;48:629-31.

35. Briceño D, Correa A, Valencia C, Torres J, Pacheco $\mathbf{R}$, Montealegre $\mathbf{M}$, et al. Actualización de la resistencia a antimicrobianos de bacilos Gram negativos aislados en hospitales de nivel III de Colombia: años 2006, 2007 y 2008. Biomédica. 2010;30:371-81.

36. Gaitán S, Espinal $\mathbf{P}$ y Grupo de Investigación en Resistencia Bacteriana, Región Caribe. Caracterización molecular de Escherichia coli y Klebsiella pneumoniae productores de $\beta$-lactamasas de espectro extendido en hospitales de la Región Caribe, Colombia. Rev Chil Infect. 2009;26:239-46. http://dx.doi.org/10.4067/S071610182009000400006

37. Secretaría Distrital de Salud de Bogotá. Área de Vigilancia en Salud Pública. Boletín Epidemiológico de Resistencia Bacteriana (SIVIBAC) 2010. Fecha de consulta: 29 de mayo de 2013. Disponible en: http://www.saludcapital. gov.co/sitios/VigilanciaSaludPublica/Paginas/IIH.aspx.

38. Grupo para el estudio de la resistencia de los antimicrobianos en Medellín. Fecha de consulta: 28 de mayo de 2013. Disponible en: http://www.grupogermen.org.

39. Grupo para el control de la resistencia bacteriana de Bogotá. Fecha de consulta: 28 de mayo de 2013. Disponible en http://www.grebo.org/grebo_site/jgrebo/index. php?option=com_content\&view=featured\&ltemid=435.

40. Red de Vigilancia Epidemiológica de Resistencia Nosocomial del Valle. Fecha de consulta: 28 de mayo de 
2013. Disponible en: http://www.renova.org.co/sitio/index. php?option=com_content\&view $=$ article\&id $=3 \& \mid$ temid $=7$

41. Villegas M, Lolans K, Correa A, Suárez C, López J, Vallejo $\mathbf{M}$, et al. First detection of the plasmid-mediated class $A$ carbapenemase KPC-2 in clinical isolates of Klebsiella pneumoniae from South America. Antimicrob Agents Chemother. 2006;50:2880-2. http://dx.doi.org/10.1128/AAC. 00186-06

42. López JA, Correa A, Navón-Venezia S, Correa AL, Torres JA, Briceño DF, et al. Intercontinental spread from Israel to Colombia of a KPC-3-producing Klebsiella pneumonia strain. Clin Microbiol Infect. 2011;17:52-6. http://dx.doi.org/10. 1111/j.1469-0691.2010.03209.x

43. Mojica M, Correa A, Vargas D, Maya M, Montealegre L, Rojas L, et al. Molecular correlates of the spread of KPCproducing Enterobacteriaceae in Colombia. Int J Antimicrob Agents. 2012;40:277-85. http://dx.doi.org/10.1016/j.ijantimicag. 2012.05.006

44. Saavedra S, Núñez J, Pulido I, González E, Valenzuela E, Reguero M, et al. Characterization of carbapenem-resistant Acinetobacter calcoaceticus-A. baumannii complex isolates in a third-level hospital in Bogotá, Colombia. Int J Antimicrob Agents.2008;31:389-91.http://dx.doi.org/10.1016/j.ijantimicag. 2007.12.008

45. Leal L, Buitrago G, Sánchez R, Castillo J, Cortés J, Álvarez C, et al. The emergence of multidrug-resistant Acinetobacter baumannii in Colombia: A time-series analysis, 2001-2007. Rev. Salud Pública. 2011;13:691-702.

46. Dellit T, Owens R, McGowan J, GerdingG, Weinstein $\mathbf{R}$, Burke J, et al. Infectious Diseases Society of America and the Society for Healthcare Epidemiology of America guidelines for developing an institutional program to enhance antimicrobial stewardship. Clin Infect Dis. 2007;44:159-77. http://dx.doi.org/10.1086/510393

47. Pallares C, Martínez E. Implementación de un programa de uso regulado de antibióticos en 2 unidades de cuidado intensivo médico-quirúrgico en un hospital universitario de tercer nivel en Colombia. Infectio. 2012;16:192-8.

48. Cataño J, Castaño O. Evaluación del impacto de un programa de vigilancia epidemiológica del consumo de antibióticos y la flora en una clínica de tercer nivel. Infectio. 2009;13:6-13

49. Venezia R, Domaracki B, Evans A, Preston K, Graffunder E. Selection of high-level oxacillin resistance in heteroresistant Staphylococcus aureus by fluoroquinolone exposure. J Antimicrob Chemother. 2001;48:375-81. http:// dx.doi.org 10.1093/jac/48.3.375

50. Rodríguez-Ganen O, Asbun-Bojalil J. Vigilancia del consumo de antimicrobianos en hospitales de México: situación actual y guía práctica para su implementación. Rev Panam Salud Pública. 2012;32:381-6.

51. Buitrago G. Relación entre el consumo de antibióticos y la resistencia bacteriana en instituciones colombianas de tercer nivel de atención (tesis). Bogotá: Universidad Nacional de Colombia; 2009. Fecha de consulta: 28 de mayo de 2013. Disponible en: http://www.bdigital.unal.edu. co/8777/1/597636.2009.pdf. 